\title{
Estimaciones del tráfico urbano de pasajeros de colectivos de Bahía Blanca, Argentina. 2007-2014
}

\section{Urban traffic estimations for bus passengers in Bahía Blanca city, Argentina. 2007-2014}

\author{
Valentina Natividad-Viego* \\ Carolina Belen-Volonté*
}

\begin{abstract}
This article aims to estimate the determinants of the demand for urban passenger transport by bus in the city of Bahia Blanca using monthly data for the 2007-2014 period. Fixed effects panel data techniques which support heterogeneity between lines are used and thus increase precision of estimates. Estimations produce elasticities (e.g. fare, cost of travel in alternative modes) which allows us to analyze the role of the general price level in the demand of bus transport.
\end{abstract}

Keywords: urban transport, bus travel demand, Bahia Blanca.

\section{Resumen}

En este artículo se realiza una estimación de los determinantes de la demanda de transporte urbano de pasajeros por colectivo de la ciudad de Bahía Blanca, para tal fin se recabaron datos mensuales del periodo 2007-2014. Se utilizan técnicas de panel de datos con efectos fijos, que admiten heterogeneidad entre líneas y mejoran la precisión de las estimaciones. La estimación permite obtener elasticidades respecto de la tarifa, del costo de desplazarse en medios alternativos y analizar el rol del nivel general de precios en el volumen de pasajeros que viajan en colectivo.

Palabras clave: transporte urbano, demanda de transporte por colectivo, Bahía Blanca. 


\section{Introducción}

La ciudad de Bahía Blanca es una localidad ubicada en el sur de la Provincia de Buenos Aires, Argentina. Si bien el departamento tiene una superficie de $2300 \mathrm{~km}^{2}$, el área urbana alcanza unos $115 \mathrm{~km}^{2} .{ }^{1}$ Según datos del último Censo Nacional de Población y Vivienda (INDEC), realizado en 2010, cuenta con una población de 300,118 habitantes en el área urbana.

La red de transporte urbano de pasajeros por colectivo está organizada en torno a un nodo central; cada uno de los recorridos que deben cumplir las diferentes líneas que prestan el servicio tienen como paso obligatorio la Plaza Rivadavia, punto central alrededor del cual se desarrolla la mayor parte de las actividades comerciales, culturales y económicas de la ciudad.

En la ciudad, la cuestión de la movilidad urbana reviste particular interés, reflejado en dos reformas en el subsistema de transporte público por colectivo que se suscitaron en 2009 y 2012 y que implicaron la fusión de líneas, modificación de recorridos, reducción de las frecuencias, modificación de sistemas de cobro, la conformación de una empresa de participación estatal mayoritaria (SAPEM) en reemplazo de un concesionario privado, entre otras. Este proceso dio como resultado, entre otros, una mayor concentración empresarial. ${ }^{2}$ Dichas reformas, a su vez, se fundaron en reclamos de las empresas prestatarias que argumentaban disminuciones severas de rentabilidad. ${ }^{3}$

En particular, la reforma de 2009 implicó la reducción de prestadores de siete a tres empresas privadas. A su vez, dos de los nuevos prestatarios eran controlados por un único grupo empresario, lo cual significaba un nivel de concentración considerablemente elevado: dicho prestador operaba $75 \%$ de las líneas de transporte público de pasajeros por colectivo. ${ }^{4}$ Luego de numerosos conflictos con los usuarios origina-

${ }^{1}$ Esta área se determina por el perímetro de las rutas que la circundan (RN 3, RP 229, RP 51, RN 252, RN 33). No se incluye la localidad de Daniel Cerri, que posee un área urbanizada de casi $4 \mathrm{~km} 2$. La delimitación de la misma, no obstante, es tentativa ya que la división entre espacios urbanos y suburbanos es difusa y dinámica en el tiempo.

${ }^{2}$ La reforma de 2012 disminuyó levemente la concentración de líneas en empresas prestatarias aunque no restauró los niveles previos a 2009.

${ }^{3}$ A fines de 2007 cuatro de los siete oferentes del servicio de transporte urbano de pasajeros (que operaban 16 de las 21 líneas operativas hasta entonces) comunicaron la interrupción en la prestación del servicio argumentando la precariedad de los contratos con el municipio (durante 40 años no fueron objeto de licitación pública), que no fijaban cláusulas de recomposición tarifaria ni revisión de las condiciones de operación ante variaciones en los costos de prestación del servicio. En 2008 dejaron de operar efectivamente tres de ellos, y a principios de 2009 se interrumpió el contrato con otras tres empresas a fin de traspasar el servicio a nuevas prestadoras.

${ }^{4}$ El pliego de licitación establecía que ninguna empresa podía licitar más de ocho líneas. La estrategia del grupo empresario que consiguió operar $75 \%$ de las líneas licitadas consistió en presentarse a la compulsa mediante una firma subsidiaria. 
dos por fallas en las condiciones de prestación del servicio -demoras en frecuencias, estado de las unidades, etcétera-, en 2012 el gobierno local estableció la caducidad del contrato con el grupo que controlaba la mayor parte del servicio. Durante el último trimestre de 2012 se produjo una nueva organización de la oferta del sistema. La prestación continuó en manos de tres empresas, una de ellas con participación mayoritaria del gobierno municipal. Desde entonces el sistema opera 17 líneas y su distribución entre prestadores es más homogénea que la situación previa. ${ }^{5}$

La gráfica 1 exhibe la evolución del volumen de pasajeros transportados durante el periodo 1996-2014. De su lectura se desprenden varios fenómenos: entre 1996 y 2002 el sistema de transporte público por colectivo experimentó una caída sostenida en los niveles transportados, la merma fue de tal magnitud que en 2002 se transportaron la mitad de pasajeros que a mediados de la década de 1990. La recuperación del nivel de actividad y consiguientemente del volumen de pasajeros transportados no consiguió alcanzar los niveles de la década previa. La brecha entre el pico de 1996-1997 y el pico del 2005-2009 es del orden de 25\% (3,000,000 vs. $2,500,000$ pasajeros). Esta merma podría atribuirse a dos posibles explicaciones, o bien, el nivel de actividad no se recuperó lo suficiente para impactar sobre el tráfico de pasajeros o, alternativamente, operó una sustitución de modos en favor del automóvil particular.

Por otro lado, enfocándonos ya en el periodo más reciente, se observa un fenómeno peculiar: desde fines de 2007 aumentó la variabilidad del número de pasajeros que se desplazan en colectivo. Entre 1996-2007, aun considerando las contracciones por la crisis de 2001-2002, la diferencia entre picos máximos y mínimos luce más o menos constante. Desde fines de 2007 se observa más inestabilidad. En particular, desde 2009 se ha alcanzado un nuevo piso inferior en el volumen de pasajeros, pasó de 2,500,000/mes durante 2005-2009 a unos 2,300,000/mes. Esta merma podría ser atribuida a la contracción experimentada en el nivel de actividad y de empleo en Bahía Blanca a partir de dicho periodo (Burachik, 2016).

En suma, en la actualidad el sistema de transporte por colectivo en la ciudad de Bahía Blanca experimenta niveles disminuidos en el tráfico de pasajeros debido tanto a cambios en la estructura de los desplazamientos (sustitución de colectivo por automóvil o ciclomotor) como a contracciones del nivel de actividad.

Como consecuencia de lo anterior, durante 2014, las autoridades municipales introdujeron distintas medidas que buscan descongestionar

${ }^{5}$ Con todo, la empresa de participación estatal mayoritaria opera menos de $20 \%$ de las líneas, mientras que las privadas retienen aún más de $80 \%$ de los recorridos. 


\section{Gráfica 1}

Bahía Blanca: Evolución de los pasajeros totales. Cifras desestacionalizadas. 1996-2014

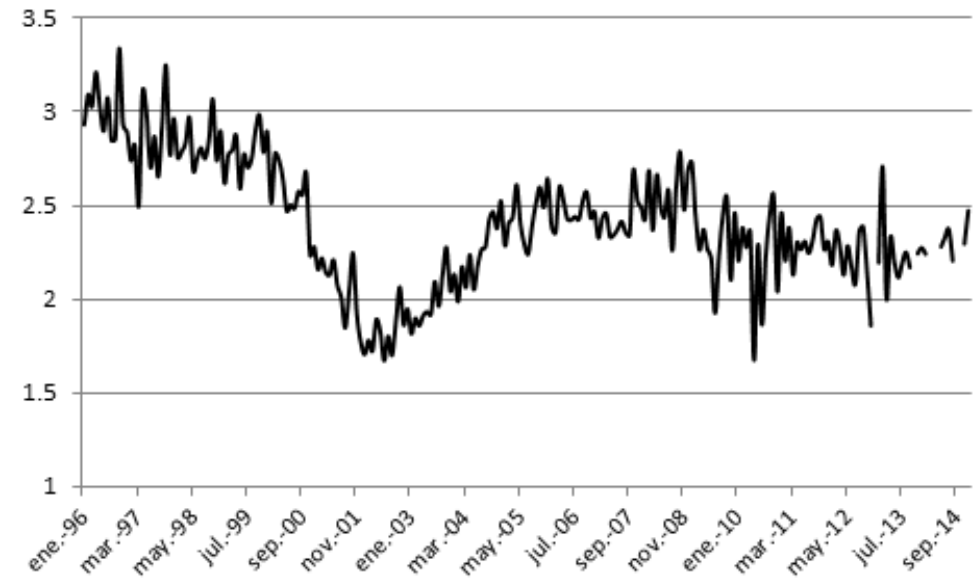

Fuente: elaboración propia en base a datos provistos por la Dirección de Tránsito y Transporte de la Municipalidad de Bahía Blanca (MBB) y difundidos por el Ministerio de Transporte de la Nación.

ciertas arterias, tales como restricciones de estacionamiento, velocidad de circulación, entre otras, desestimulando el uso del automóvil particular, medidas que a su vez han generado reacciones diversas entre los residentes y los comerciantes.

\section{Objetivo}

En el presente trabajo se mide la contribución de ciertos determinantes de la demanda de transporte público de pasajeros por colectivo para la ciudad de Bahía Blanca. En particular se busca identificar el rol de las tarifas del servicio sobre el volumen transportado con el fin de enriquecer la comprensión del fenómeno, revisar medidas adoptadas en el sector y ofrecer elementos para la toma de decisiones en el futuro.

\section{Revisión de la literatura}

Existe una variada literatura aplicada dedicada al estudio del transporte urbano de pasajeros. La mayor parte se focaliza en ciudades de países desarrollados. Los estudios aplicados varían en metodología según el 
objetivo del análisis y según la disponibilidad y grado de desagregación de los datos.

En los países en desarrollo o centros urbanos no metropolitanos (menos de un millón de habitantes) los métodos aplicados suelen basarse más en datos temporales que de corte transversal debido al mayor costo de realizar relevamientos para indagar sobre disposición a pagar, modos de transporte empleados, etcétera.

A su vez, la mayor parte de los análisis suelen centrarse en estimar elasticidades precio de la demanda, es decir, las variaciones en los niveles transportados ante variaciones en la tarifa del servicio. En particular, las referencias que ofrecen cálculos de elasticidad precio del transporte urbano por colectivo se ubican en valores entre -0.1 y -0.8 (ver recopilación de Orro Arcay et al., 2002). La revisión de estudios realizada por Wardman y Shires (2003) para distintas ciudades del Reino Unido muestra que la elasticidad precio promedio del transporte en colectivo se ubicaría en torno a -0.5. La recopilación de Webster y Bly (1980) ubica el valor promedio en torno a -0.3. La diferencia podría explicarse según el horizonte temporal considerado (corto plazo vs. largo plazo).

Los niveles estimados dependen del tamańo de la ciudad, elasticidades mayores en ciudades más pequeñas y viceversa, y de la magnitud en que varían las tarifas. ${ }^{6}$ Con todo, Gschwender y Jara-Díaz (2007) advierten sobre la relativa estabilidad de los valores de elasticidad de la demanda respecto de la tarifa de colectivo.

En el ámbito nacional, los antecedentes son escasos debido a la falta de datos oficiales o generados por los propios investigadores (mediante relevamientos). Se han realizado estudios con datos primarios sobre determinantes de la demanda de transporte urbano en las ciudades de Salta y Posadas (Liendro, 2013) y Gran Mendoza (Reta, 2006). Sin embargo, dichos estudios estiman los determinantes de la elección del modo de desplazamiento dentro de la ciudad (colectivo, automóvil particular, taxi, etc.) sin referirse al rol de los precios.

Con relación a elasticidades, Sartori (2003) realiza estimaciones de elasticidades de corto plazo para la ciudad de Córdoba para el periodo 1993-2000 en torno a -0.29. La referencia más reciente es Montalvo (2015) para la ciudad de Buenos Aires con modelos dinámicos que permiten obtener elasticidades de corto y largo plazo. Los valores obtenidos por dicho autor para la elasticidad precio de largo plazo del transporte por colectivo son similares a los del Reino Unido (cercanos a -0.5). Con todo, ambos trabajos enfrentan algunas limitaciones metodológicas.

\footnotetext{
${ }^{6}$ La elasticidad precio también difiere en distintas horas del día, por ejemplo, en las horas pico es menor que en el resto de los tramos horarios.
} 
En primer lugar utilizan datos para el total de líneas de colectivo, perdiendo la riqueza que podría aportar información desagregada por línea o, al menos, por prestador. Además, emplean modelos de doble logaritmo, que suponen estabilidad en las elasticidades respecto de los precios e indicadores del nivel de actividad cuando durante el periodo analizado en ambas ciudades se experimentaron transformaciones económicas, sociales y territoriales que probablemente afectaron la sensibilidad de los volúmenes de pasajeros a los precios del servicio y de otras alternativas.

Además, en el caso de Montalvo (2015) se estima un modelo dinámico autorregresivo, herramienta que permite obtener elasticidades de corto y largo plazo, pero no se examina la presencia de autocorrelación serial en los residuos, fenómeno que volvería inconsistentes a los estimadores. Este autor no encuentra en la función de demanda de transporte por colectivo cointegración, es decir, no una relación de largo plazo entre las variables probablemente debido a la omisión de variables relevantes, como el stock de vehículos particulares o indicadores del costo de desplazamiento en automóvil. Estas limitaciones, de algún modo, limitan el alcance y validez de esos resultados.

\section{Metodología y datos}

La metodología empleada se basa en la estimación de una función de demanda utilizando la técnica de panel de datos con efectos fijos. Para llevar a cabo dichas estimaciones se emplearon datos correspondientes al periodo 2007-2014, con periodicidad mensual. ${ }^{7}$ La extensión del periodo se determinó en función de la disponibilidad de datos. Por otro lado, coincide con el periodo de mayor volatilidad en el volumen de pasajeros señalada anteriormente.

La función de demanda a estimar adopta la siguiente especificación:

$$
Q_{i t}=\alpha+\sum_{k=1}^{k} \beta_{k} \ln X_{k i t}+u_{i t} \text { con } i: 1, \ldots, 15 \mathrm{y} t .1, \ldots, 96
$$

donde $Q_{i t}$ es el número de pasajeros totales de la línea de colectivo $i$, en el mes $t . X_{k}$ es una matriz que reúne a las variables explicativas consideradas, en este caso se incluyen la tarifa de colectivo $(T A R)$, la tarifa de $\operatorname{taxi}^{8}(T A X)$, el número de patentamientos de autos $0 \mathrm{~km}$ en la ciudad (PAT), que opera como proxy del parque de automóviles particulares

\footnotetext{
${ }^{7}$ Se debe aclarar que los cambios en la estructura de la oferta del sector no provocaron desbalances en el panel, en tanto afectaron a los operadores y no a las líneas.

${ }^{8}$ La tarifa del transporte en taxi tiene dos componentes: bajada de bandera, que es una suma fija por cada viaje realizado y un monto variable por cada 100 metros recorridos. La evolución de ambos componentes ha sido similar. Se optó por emplear el componente variable.
} 
existente, el precio de la nafta $(P N A F)$ y el nivel general de precios $(I P C)$. En particular, la inclusión de PAT y de PNAF da cuenta del rol de automóvil particular como modo de transporte en las ciudades, elementos que no fueron incluidos en estudios empíricos previos (Sartori, 2003, Montalvo, 2015) para el caso de Argentina.

Se espera de que la tarifa de colectivo y el parque automotor incidan negativamente sobre el volumen transportado y el costo de desplazarse en taxi y que el precio del combustible que utilizan mayormente los automóviles ejerzan un efecto positivo sobre la cantidad de pasajeros del sistema de colectivos. Respecto del efecto del nivel general de precios, hay dos efectos de signo contrario. Por un lado, el aumento generalizado de precios disminuye el poder adquisitivo de los hogares, tiene efectos recesivos y desalienta el nivel de actividad; lo cual se expresaría en un coeficiente de signo negativo. Por otro lado, la merma en la capacidad de compra del ingreso puede dar lugar a una sustitución entre medios de transporte, desalentando el uso del automóvil a favor del colectivo. Por ello, el signo esperado del coeficiente que registra la tasa inflacionaria es ambiguo.

Además se incluyó una tendencia temporal y una variable binaria $(G R U)$ que toma valor unitario en las líneas y periodo en que operó el grupo empresario que concentró 75\% de las líneas durante 2008-2012. $\beta$ es el vector de los parámetros a estimar. El subíndice $i$ representa a cada una de las líneas en funcionamiento en la localidad, $t$ hace referencia al periodo, en este caso expresado mensualmente.

Por último, $u_{i t}$ es el término de error. En los modelos de panel de datos con efectos fijos, el término de perturbación tiene dos componentes: $u_{i t}=\alpha_{i}+\varepsilon_{i t}$ donde $\alpha_{i}$ es un componente variable en i pero invariante en el tiempo, que capta la heterogeneidad de corte transversal, mientras que $\varepsilon_{i t}$ es un término i.i.d. En este caso, $\alpha_{i}$ contiene especificidades de cada línea no contempladas en los regresores (e.g. densidad poblacional en el recorrido realizado, perfil de pasajeros, etc.).

Conviene recordar que, como el modelo adopta una especificación lin-log los parámetros $\beta$ se interpretan como semi-elasticidades (cambios en cantidad de pasajeros ante cambios porcentuales en cada regresor). $\mathrm{Si}$ bien en los estudios empíricos que estiman elasticidades de la función de demanda de transporte urbano es más frecuente encontrar el uso de especificaciones de doble logaritmo, esto tiene el inconveniente de suponer elasticidades precio constantes para cualquier nivel de tarifa. Además, en el caso particular de datos de panel, implicaría suponer que cada línea tiene elasticidades iguales, factor que no necesariamente se cumple considerando que en el sistema funcionan líneas con distinto perfil de recorrido, sirviendo a barrios con distinto nivel socioeconómico y posibilidades de sustitución entre medios de transporte. 
En Bahía Blanca, la tarifa de colectivo se multiplicó por cinco durante el periodo estudiado, hecho que avalaría el empleo de especificaciones que admitan variaciones en la elasticidad precio y, por ende, una estimación semi-log. Además, las estimaciones doble log mostraron un peor ajuste, reflejado en mayores valores de los criterios de información de Akaike y de Schwartz, en los datos locales respecto de la variante lin-log. Litman (2004) reconoce el empleo de especificaciones semi-log indica que ambas especificaciones arrojan resultados similares en tanto las tarifas se encuentren en un rango de variación entre 10\%-30\%. En el periodo analizado en Bahía Blanca cada incremento tarifario se ubicó en torno a $22 \%$, guarismo ubicado en el intervalo donde ambas especificaciones no difieren sustancialmente.

Por otro lado, se consideraron dos definiciones sobre la variable dependiente. Por un lado, la cantidad de pasajeros totales transportados y, por otro, la cantidad de pasajeros que abonaron la tarifa plena sin ningún tipo de descuento, suponiendo que los parámetros pueden diferir según el tipo de usuario del servicio.

Se dispone de información de 22 líneas desde enero de 2007 a diciembre de 2014. No obstante, el panel no está balanceado ya que varias líneas no operaron durante el periodo considerado y hay datos faltantes para algunos de los últimos meses de 2014. Se controlaron los efectos de este desbalance en el panel sobre los resultados. Las estimaciones fueron realizadas empleando el paquete econométrico Stata v 12.

En la tabla 1 se detallan los datos empleados y las fuentes de información.

La ventaja del método utilizado respecto de las técnicas empleadas en los estudios citados es la de admitir la heterogeneidad de líneas, capturada a través del término $\alpha_{i}$, que registra los niveles de pasajeros (totales y sin descuento) que cada línea transporta con independencia de los precios y parque automotor. ${ }^{9}$ Además, la especificación semi-logarítmica admite variaciones de las elasticidades precio y cruzadas variantes tanto por línea como en el tiempo; elemento que agrega riqueza al análisis.

Se reconoce, no obstante, que el desbalance del panel impide analizar si los modelos propuestos cointegran a las variables involucradas; las herramientas disponibles que chequean la existencia o no de resultados espurios (test de Pedroni o de Westerlund, por citar algunos) requieren paneles balanceados. Por este motivo, no es posible establecer con algún grado de confianza si las relaciones estimadas entre las variables son de largo plazo.

\footnotetext{
${ }^{9}$ A su vez, estos factores no observables podrían estar asociados a las rutas cubiertas por cada línea, que pueden involucrar distintos perfiles socioeconómicos de la población servida. Un relevamiento de usuarios del sistema podría contribuir a corroborar si la cantidad de pasajeros de base de cada línea puede estar asociada a estos factores.
} 


\section{Tabla 1}

Definición de variables empleadas y fuentes de información

\begin{tabular}{|c|c|c|}
\hline Variable & Definición & Fuente de información \\
\hline \multicolumn{3}{|l|}{ Dependiente } \\
\hline$Q(1)$ & pasajeros totales mensuales & $\begin{array}{l}\text { Datos facilitados por la Dirección } \\
\text { de Tránsito y Transporte de la } \\
\text { Municipalidad de Bahía Blanca }\end{array}$ \\
\hline$Q(2)$ & $\begin{array}{l}\text { pasajeros mensuales que abonaron la } \\
\text { tarifa total }\end{array}$ & $\begin{array}{l}\text { Datos facilitados por la Dirección } \\
\text { de Tránsito y Transporte de la } \\
\text { Municipalidad de Bahía Blanca }\end{array}$ \\
\hline \multicolumn{3}{|l|}{ Explicativas } \\
\hline$T A R$ & $\begin{array}{l}\text { tarifa plana correspondiente a la } \\
\text { primera sección, en pesos }\end{array}$ & $\begin{array}{l}\text { Diario La Nueva Provincia, varias } \\
\text { notas }\end{array}$ \\
\hline PAT & $\begin{array}{l}\text { automóviles } 0 \mathrm{~km} \text { patentados en } \\
\text { Bahía Blanca }\end{array}$ & $\begin{array}{l}\text { Centro Regional de Estudios } \\
\text { Económicos de Bahía Blanca } \\
\text { Argentina (CREEBBA). }\end{array}$ \\
\hline TAX & $\begin{array}{l}\text { tarifa del taxímetro cada } 100 \text { metros } \\
\text { recorridos, en pesos }\end{array}$ & $\begin{array}{l}\text { Diario La Nueva Provincia, varias } \\
\text { notas }\end{array}$ \\
\hline PNAF & $\begin{array}{l}\text { precio de nafta súper ( } 92-95 \text { Ron) en } \\
\text { estaciones de servicio YPF }\end{array}$ & $\begin{array}{l}\text { Ministerio de Energía y Minería, } \\
\text { Res. } 1104 / 2004\end{array}$ \\
\hline$I P C$ & $\begin{array}{l}\text { Índice de precios al consumidor base } \\
2002=100 \text { en Bahía Blanca }\end{array}$ & $\begin{array}{l}\text { Centro Regional de Estudios } \\
\text { Económicos de Bahía Blanca } \\
\text { Argentina (CREEBBA, 2016) }\end{array}$ \\
\hline$G R U$ & $\begin{array}{l}=1 \text { si la línea es operada por Grupo } \\
\text { empresario y } 0 \text { en caso contrario. }\end{array}$ & \\
\hline
\end{tabular}

Fuente: elaboración propia.

\section{Resultados}

Las estimaciones realizadas tanto para pasajeros totales como los que abonaron la tarifa completa indican que un incremento del uno por ciento en el precio del boleto provocaría una disminución del volumen de pasajeros mensuales entre $0.06 \%$ y $0.35 \%$, aproximadamente. ${ }^{10} \mathrm{El}$ efecto del precio del taxi sobre el volumen de pasajeros mensuales es algo más amplio que el del precio del boleto, además de tener el signo contrario; por cada incremento del uno por ciento en el costo de desplazamiento en taxi el sistema de colectivos atraería pasajeros en una proporción promedio de $0.22 \%$. La magnitud de ambos efectos no difiere sustancialmente tanto si la dependiente se define como pasajeros totales o sólo considera a los que

${ }^{10}$ Este guarismo surge de tomar los valores del intervalo de confianza correspondiente a la tarifa multiplicados por 22 (cantidad de líneas) y dividido por el volumen total de pasajeros del sistema. 
no fueron beneficiados con descuentos. Tampoco se registran cambios sustanciales cuando el modelo es estimado omitiendo los datos de las seis líneas que tuvieron discontinuidades durante el periodo (tabla 2).

El resto de las variables consideradas exhibe distintos resultados según se consideren los pasajeros totales o los que abonaron la tarifa completa. Cuando la dependiente equivale a los pasajeros totales, se verifica una merma de entre $0.03 \%$ y $0.13 \%$ de los desplazamientos mensuales en colectivo ante incrementos del uno por ciento en el parque automotor. Respecto de la tasa de variación de precios, se registra que mayores niveles inflacionarios favorecen la sustitución desde los desplazamientos en automóvil hacia el sistema público por colectivo. Estos efectos no resultan significativos cuando la dependiente sólo considera a los pasajeros que abonaron la tarifa plena probablemente debido a la multicolinealidad entre regresores (tabla 3).

No hay resultados concluyentes sobre los efectos que tuvo sobre el tráfico de pasajeros la concesión de $75 \%$ de las líneas a un grupo empresario durante 2009-2012; en el caso de los pasajeros totales, no se registran variaciones significativas en la demanda. Sin embargo, se observa aumento de la demanda del orden de $0.02 \%$ y $0.09 \%$ boletos por mes cuando se considera sólo el volumen de pasajeros que abonaron la tarifa completa en las líneas operadas por el grupo empresario.

La especificación utilizada (lin-log) admite variaciones temporales en las elasticidades de la demanda respecto de cada una de las variables explicativas consideradas. Dichas elasticidades se obtienen dividiendo a cada coeficiente significativo estimado por el nivel absoluto de la dependiente (premultiplicado por 100). Se trata, además de elasticidades de corto plazo, debido a la frecuencia de los datos empleados. ${ }^{11}$ Considerando el conjunto de 22 líneas y 90 periodos analizados, ${ }^{12}$ la elasticidad precio de corto plazo promedio es de -0.21 en el caso de pasajeros totales y -0.25 en los pasajeros sin descuentos. Estos valores son algo inferiores a la estimación obtenida por Sartori (2003) para Córdoba. Esto se puede explicar por el hecho de que en esa ciudad hay mayores posibilidades de sustitución entre medios de transporte público (trolebús). Además, se debe recordar que la elasticidad reportada aquí es una cifra promedio para el conjunto de líneas que operan el sistema. Al utilizar un modelo semi-log la elasticidad precio de cada línea no necesariamente coincide con la global $(0.12$ y decreciente en el caso de la línea 500 vs. 0.35 y en alza en la línea 509). Por su parte, las elasticidades de la demanda respecto de la tarifa de taxi

\footnotetext{
${ }^{11}$ La obtención de elasticidades de largo plazo requeriría mayor volumen de datos por línea para poder emplear técnicas basadas en relaciones de cointegración.

${ }^{12}$ No se cuenta con datos para el periodo completo de 96 meses que va de enero de 2007 a diciembre de 2014 debido a datos faltantes en algunos meses de 2014 .
} 


\section{Tabla 2}

\section{Transporte público de pasajeros de Bahía Blanca. Panel de datos con efectos fijos 2007-2014}

\begin{tabular}{|c|c|c|}
\hline & $\begin{array}{c}\text { Q1 } \\
\text { pasajeros totales }\end{array}$ & $\begin{array}{c}Q 2 \\
\text { pasajeros tarifa plena }\end{array}$ \\
\hline $\ln T A R, \beta_{1}$ & $\begin{array}{c}-21389.6^{* * *} \\
(7573.3) \\
{[-6534.4 ;-36244.7]}\end{array}$ & $\begin{array}{c}-21326.2^{* * *} \\
(-6853.3) \\
{[-7883.3 ;-34769.1]}\end{array}$ \\
\hline $\ln T A X, \beta_{2}$ & $\begin{array}{c}23230.3^{* * *} \\
(9774.8) \\
{[4056.8 ; 42403.8]}\end{array}$ & $\begin{array}{c}18033.3^{* * *} \\
(8845.5) \\
{[682.6 ; 35383.9]}\end{array}$ \\
\hline $\ln P A T, \beta_{3}$ & $\begin{array}{c}-8680.1^{* * *} \\
(2653.2) \\
{[-3475.7 ;-13884.5]}\end{array}$ & $\begin{array}{c}1093.8 \\
(2401.0)\end{array}$ \\
\hline $\ln P N A F, \beta_{4}$ & $\begin{array}{l}-3859.2 \\
(10870)\end{array}$ & $\begin{array}{c}8235.5 \\
(9836.6)\end{array}$ \\
\hline $\operatorname{Ln} I P C, \beta_{5}$ & $\begin{array}{c}3282.9^{* *} \\
(1553.71) \\
{[235.2 ; 6330.5]} \\
\end{array}$ & $\begin{array}{c}1188.6 \\
(1406.0)\end{array}$ \\
\hline$G R U, \beta_{6}$ & $\begin{array}{l}-1853.9 \\
(2253.1)\end{array}$ & $\begin{array}{c}5682.7^{* * *} \\
(2038.9) \\
{[1683.4 ; 9682.0]}\end{array}$ \\
\hline Constante, $\beta_{0}$ & $\begin{array}{c}213698.9^{* * *} \\
(33513.8) \\
{[147960.8 ; 279437]}\end{array}$ & $\begin{array}{c}141078.6^{* * *} \\
(30327.7) \\
{[81590.2 ; 200567.1]}\end{array}$ \\
\hline No. Observaciones & 1553 & 1553 \\
\hline $\begin{array}{l}\text { Test efectos fijos no } \\
\text { significativos, } \alpha_{i}=0 \\
\text { F }\end{array}$ & $163.12^{* * *}$ & $144.9^{* * *}$ \\
\hline
\end{tabular}

( ) errores estándar entre paréntesis.

*** Significativas al 1\%. ${ }^{* *}$ significativas al $5 \%$. ${ }^{*}$ significativas al $10 \%$.

Intervalos de confianza al $95 \%$ entre corchetes

Fuente: elaboración propia.

Tabla 3

Matriz de correlación entre regresores

\begin{tabular}{|l|c|c|c|c|c|}
\hline & $\ln T A R$ & $\ln T A X$ & $\ln P A T$ & $\ln P N A F$ & $\ln I P C$ \\
\hline $\ln T A R$ & 1 & & & & \\
\hline $\ln T A X$ & 0.9608 & 1 & & & \\
\hline $\ln P A T$ & 0.3202 & 0.3711 & 1 & & \\
\hline $\ln P N A F$ & 0.9650 & 0.9815 & 0.3157 & 1 & \\
\hline $\ln I P C$ & 0.6222 & 0.6486 & 0.2337 & 0.6577 & 1 \\
\hline
\end{tabular}

Fuente: elaboración propia. 
son algo menores en pasajeros totales y algo mayores en pasajeros sin descuento.

A fin de ilustrar los resultados, se calcularon las elasticidades precio del volumen de pasajeros en cinco líneas seleccionadas (gráfica 2): la línea que transporta más pasajeros en promedio (línea 517), una línea con un volumen intermedio de pasajeros mensuales (línea 506), y la línea que menor cantidad de pasajeros traslada en promedio (línea 509). Se calcularon además las elasticidades de las principales líneas operadas por cada empresa (línea 517 que, a su vez, resulta ser la más voluminosa; línea 502 y línea 500).

En general, se observa que las elasticidades precio de los pasajeros totales son menores a la de los pasajeros que abonan la tarifa completa del servicio y que dicha brecha ha tendido a ampliarse, especialmente desde 2011. El resultado es lógico en tanto los pasajeros que acceden a descuentos en la tarifa suelen viajar en horarios pico, con mejor elasticidad que los viajes realizados fuera del horario pico. Además, si no se otorgaran descuentos, los aumentos de la tarifa darían lugar a reducciones en la cantidad de viajes tanto por contracción de los desplazamientos totales como por sustitución de modos de transporte. En general, los aumentos tarifarios impactan menos sobre los pasajeros que gozan de descuentos y la contracción en ese segmento suele ocurrir más por cambio de modo antes que por reducción en el número de viajes (White, 1981).

No obstante, se observa que la magnitud de las elasticidades es sustancialmente menor a la registrada en otras regiones (incluso similares en otras ciudades de Argentina como Córdoba o Ciudad Autónoma de Buenos Aires). La explicación de esto es que las elasticidades precio estimadas por otros estudios no distingue entre líneas, sino que analiza la demanda total del sistema. En nuestro caso, como cada línea compite sólo parcialmente por pasajeros (debido a que las áreas servidas son distintas y pueden solaparse sólo cerca del microcentro), la demanda cautiva de cada línea adquiere un mayor peso, reduciendo las elasticidades. En este caso, los valores se acercan a los proporcionados por Webster y Bly (1980) para pasajeros cautivos (en torno a -0.1).

\section{Discusión}

Si bien la metodología empleada admite heterogeneidad entre observaciones de corte transversal, en este caso líneas, no se dispone de información sobre la calidad del servicio de cada línea (en términos del tiempo de espera promedio en la parada, confort de las unidades, tiempo de viaje, etc.). Con base en relevamientos a individuos, Matas (1991) estima que los individuos resultan menos sensibles a los precios que a la calidad. 


\section{Gráfica 2}

Elasticidad precio de la demanda de transporte por colectivo. Líneas seleccionadas, 2007-2014

a) Línea 500
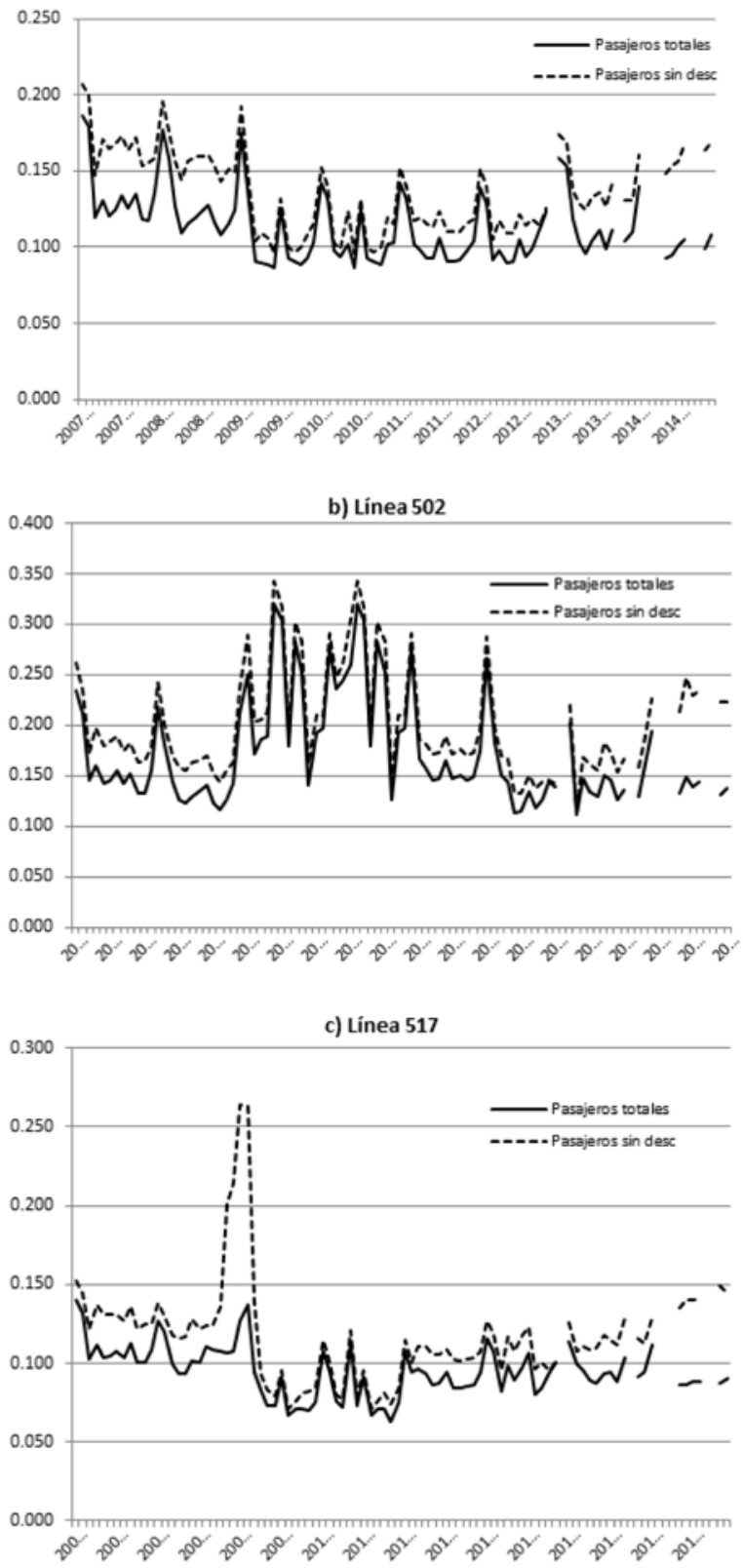

Fuente: elaboración propia con base a datos provistos por la Dirección de Tránsito y Transporte de la MBB (2015). 
Gráfica 2 (continuación)

d) Línea 506

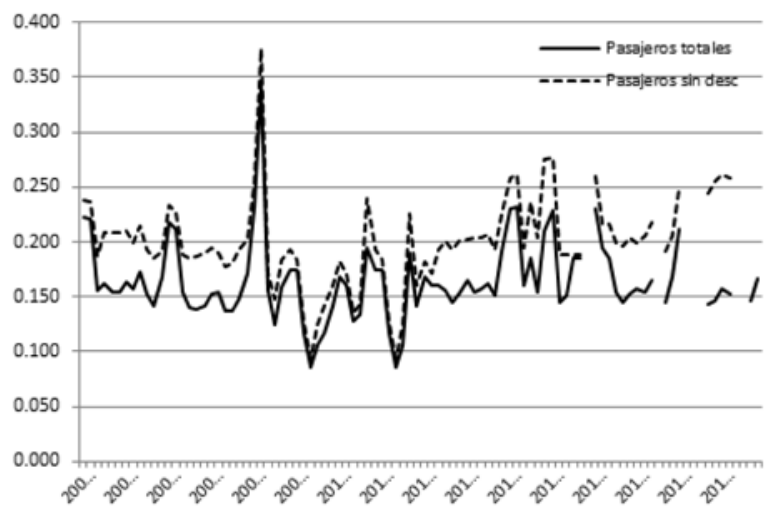

e) Línea 509

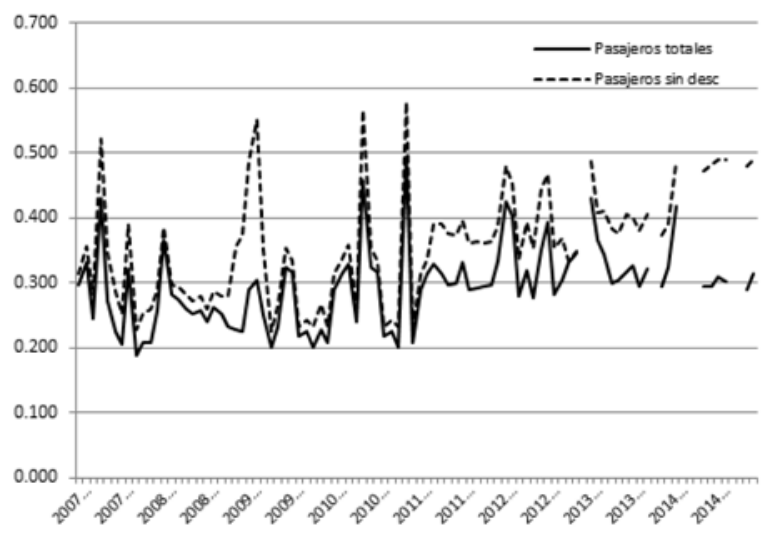

Fuente: elaboración propia con base a datos provistos por la Dirección de Tránsito y Transporte de la MBB (2015).

Por otro lado, los datos no permiten discriminar elasticidades precio según el nivel socioeconómico de los usuarios; el mismo estudio de Matas, citado anteriormente, muestra que la elasticidad precio de los usuarios menos calificados es menor que la de los usuarios de alta calificación. Esto se debe a la posibilidad de sustitución entre modos de transporte, acotada para los usuarios de menor nivel socioeconómico.

En relación con lo anterior, Matas (1991) recomienda que las políticas tendentes a reducir la congestión y, en general, la gestión del tráfico se hagan recurriendo a peajes en vez de subsidiar la tarifa de colectivos. La aplicación de este criterio en áreas urbanas de países subdesarrollados, como el caso aquí analizado, tiene dos consecuencias especialmente 
negativas: por un lado, implica un aumento neto del gasto privado de los hogares (en tanto se reducirían los subsidios al transporte por bus al tiempo que aumentarían los pagos de conductores de vehículos particulares). Como bien reconoce Matas (1991), la menor sensibilidad de los automovilistas a los precios implicaría que, para ser efectivos con las metas de reducción de tránsito, los peajes deberían ser elevados. ${ }^{13}$ Segundo, una política basada en impuestos que penalicen la movilidad privada perjudicaría a los hogares con baja conexión a la red de transporte público o aquellos que contando con acceso cercano al bus, utilizan vehículo particular debido a déficit en la prestación del servicio público (pocas frecuencias, incertidumbre respecto de horarios, etc.). En suma, para que los gobiernos locales recurran a políticas que gravan el uso del automóvil particular, es necesario que primero se fortalezca la oferta de transporte público de modo que éste alcance la condición de sustituto genuino del modo privado. En Bahía Blanca, al igual que otras ciudades del interior del país, la sustituibilidad entre la movilidad privada y pública es aún limitada.

\section{Conclusiones}

En Bahía Blanca, la evolución del número de pasajeros transportados por colectivo muestra una contracción global que data desde mediados de la década de 1990. Si bien la salida de la crisis económica de 2002 permitió recuperar los volúmenes transportados, los niveles no alcanzaron los picos previos. Los máximos niveles de pasajeros en cada periodo de auge económico (1996-1997 vs. 2005-2009) difieren en 25\%, lo que podría atribuirse a una recuperación económica insuficiente para impulsar los desplazamientos en colectivo o una progresiva sustitución de modos a favor del automóvil particular.

A su vez, desde 2007 el sistema se encuentra expuesto a una mayor variabilidad en los volúmenes de pasajeros transportados, que podría vincularse a los problemas de rentabilidad de los operadores, los que al encontrar obstáculos para variar las tarifas en el corto plazo, alteran la calidad del servicio (menores frecuencias, contracción de gastos de mantenimiento de unidades que redundan en roturas, etc.). A ello se suman

\footnotetext{
${ }^{13}$ Matas (1991) dispone de datos primarios recogidos en una encuesta donde se distingue si el usuario utiliza transporte público y privado. Ello le permite estimar la sensibilidad de pasajeros de transporte público y privado a distintas dimensiones (precio, tiempo de viaje, etc.). En nuestro caso, no contamos con datos para contrastar en el caso local si los usuarios de vehículos particulares son menos sensibles a los precios que los que se desplazan en colectivo.
} 
dos reformas en el sistema de concesión que derivaron en una mayor concentración empresarial.

El artículo estima el rol de la tarifa de colectivo, del costo de desplazarse en taxi, del costo del combustible, del parque automotor y de la evolución del nivel general de precios sobre los volúmenes transportados con un modelo de datos de panel con efectos fijos diferenciando entre pasajeros totales y pasajeros que no gozaron de descuentos tarifarios.

Los resultados indican que la elasticidad precio de la demanda de transporte en colectivo se ubica en torno a -0.21 y -0.25 , según se consideren pasajeros totales o sólo los que abonan el precio completo del boleto. Sin embargo, estos guarismos varían en el tiempo y entre líneas. Desde 2011 en particular se registra cierta alza en la elasticidad precio de los pasajeros sin descuentos. A su vez, las líneas con menor tráfico exhiben mayores elasticidades precio.

A su vez, los pasajeros que no tienen descuentos en el viaje en colectivo son menos sensibles al costo de desplazarse en taxi que el resto. El efecto del resto de las variables se encuentra oculto debido a la elevada correlación entre los regresores. De todos modos, se alcanza a registrar significación del parque automotor y de la variación del nivel general de precios en la demanda de desplazamientos en colectivo.

La posibilidad de robustecer las estimaciones depende críticamente de la disponibilidad de datos, lo cual requiere del fortalecimiento del sistema de control y de la generación de información primaria sobre el perfil de usuarios de cada línea con el fin de estimar impactos de medidas tarifarias, fusión de líneas, tiempos de espera, entre otras, en distintos estratos socioeconómicos.

\section{Fuentes consultadas}

Burachik, Gustavo (2016), "Empleo y desempleo en Bahía Blanca en los años 2003-2013”, Estudios Económicos, 32 (64), Departamento de Economía, Universidad Nacional del Sur, Bahía Blanca, Argentina, pp. 83-100.

CREEBBA (Centro Regional de Estudios Económicos de Bahía Blanca Argentina) (2016), "INFORMES IPC Planilla Serie IPC", CREEBBA, Bahía Blanca, Argentina, <http://www.creebba.org. ar/movil_new/ipc/informe_ipc.php>, 12 de julio de 2016.

Diario La Nueva Provincia (2008a), "Se confirmó un aumento del 28\% en la tarifa de taxis", Diario La Nueva Provincia, 9 de mayo, Difusión S. A., Bahía Blanca, Argentina, p. 6. 
Diario La Nueva Provincia (2008b), "Rige desde hoy el aumento del boleto de ómnibus", Diario La Nueva Provincia, 7 de noviembre, Difusión S. A., Bahía Blanca, Argentina, p. 7.

Diario La Nueva Provincia (2009a), "El Concejo aprobó anoche el aumento del boleto a 1,75", Diario La Nueva Provincia, 29 de mayo, Difusión S. A., Bahía Blanca, Argentina, p. 5.

Diario La Nueva Provincia (2009b), "El Concejo Deliberante aprobó aumento del $13 \%$ en la tarifa de taxis", Diario La Nueva Provincia, 9 de junio, Difusión S. A:, Bahía Blanca, Argentina, p. 6.

Diario La Nueva Provincia (2010), "El decreto de aumento del boleto no llegó al Concejo", Diario La Nueva Proviencia, 2 de setiembre, Difusión S. A., Bahía Blanca, Argentina, p. 7.

Diario La Nueva Provincia (2011), "El CD aprobó un aumento para servicio de taxis", Diario La Nueva Provincia, 20 de agosto, Difusión S. A., Bahía Blanca, Argentina, p. 8.

Diario La Nueva Provincia (2012), "Desde la hora 0 de mañana comenzará a regir la nueva tarifa del servicio de taxis", Diario La Nueva Provincia, 9 de junio, Difusión S. A., Bahía Blanca, Argentina, p. 5.

Diario La Nueva Provincia (2013), "El servicio de taxi tiene nuevos costos", Diario La Nueva Provincia, 10 de diciembre, Difusión S. A., Bahía Blanca, Argentina, p. 6.

Diario La Nueva Provincia (2014), "La suba del colectivo impacta más a los pasajeros frecuentes", Diario La Nueva Provincia, 3 de diciembre, Difusión S. A., Bahía Blanca, p. 8.

Dirección de Tránsito y Transporte (2015), "Pasajeros transportados por línea de colectivo, totales, según tipo de tarifa abonada, 20072014, frecuencia mensual”, Municipalidad de Bahía Blanca, Buenos Aires, Argentina.

Gschwender, Antonio y Sergio Jara-Díaz (2007), "Elasticidades de la demanda del transporte público urbano: síntesis e interrelaciones", Ingeniería de Transporte, 13 (1), Sociedad Chilena de Ingeniería de Transporte, Santiago, Chile, pp. 5-10. 
INDEC (Instituto Nacional de Estadística y Censos) (2010), "Censo Nacional de Población y Vivienda 2010”, INDEC, Buenos Aires, Argentina, <http://www.indec.gov.ar/bases-de-datos. asp?solapa 5>, 15 de febrero de 2016.

Liendro, Nicolas (2013), "Determinantes de la demanda de transporte: una comparación entre Salta y Posadas", ponencia presentada en la XLVIII Reunión Anual Asociación Argentina de Economía Política, Facultad de Ciencias Económicas y Estadística, Universidad Nacional de Rosario, del 13 al 15 de noviembre, Rosario, Argentina.

Litman, Todd (2004), "Transit Price elasticities and cross elasticities", Journal of Public Transportation, 7 (2), National Center for Transport Research, South Florida, Estados Unidos de América, pp. 37-58.

Matas, Anna (1991), "La demanda de transporte urbano: un análisis de las elasticidades y valoraciones del tiempo", Investigaciones Económicas, 15(2), Universidad Autónoma de Barcelona, Barcelona, España, pp. 249-267.

Ministerio de Energía y Minería (2016), "Resolución SE 1104/2004", Ministerio de Energía y Minería, Buenos Aires, Argentina, $<$ http://www.energia.gob.ar/contenidos/verpagina.php_idpagina $=2576>, 23$ de marzo de 2016.

Montalvo, Jerónimo (2015), "Elasticidad-precio de la demanda del transporte público urbano: un análisis para los servicios de ómnibus y subterráneo de la Ciudad Autónoma de Buenos Aires", tesis de maestría, Universidad Nacional de la Plata, Argentina, <http:// blogs.eco.unc.edu.ar/jifp/files/t23.pdf>, 23 de febrero de 2016.

Orro-Arcay, Alfonso, Miguel Rodríguez-Bugarin, Margarita NovalesOrdax y Mar Chao-Lopez (2002), "Influencia de las variaciones en las tarifas del autobús en el transporte urbano", V Congreso de Ingeniería del Transporte, Universidad de Cantabria, del 11 al 13 de junio, Santander, España, <http://caminos.udc.es/grupos/ ferroca/orro/documentos/elast_orro_CIT2002.pdf>, 4 de marzo de 2016.

Reta, Cintia (2006), "Determinantes de la demanda de transporte urbano en el Gran Mendoza; encuesta origen destino 2005", ponencia 
presentada en la XLI Reunión Anual de la Asociación Argentina de Economía Política, Universidad Nacional de Salta, del 11 a 14 de noviembre, Salta, Argentina.

Sartori, Juan José (2003), "Estimación de las elasticidades de demanda para el transporte urbano de la ciudad de Córdoba (Argentina)", ponencia presentada en la XXXVIII Reunión Anual de la Asociación Argentina de Economía Política, Universidad Nacional de Cuyo, del 11 al 14 de noviembre, Mendoza, Argentina.

Wardman, Mark y Jeremy Shires (2003), "Review of the fare elasticities in Great Britain", Working Paper 573, Institute of Transport Studies, Leeds, Inglaterra, <http://eprints.whiterose.ac. uk/2059/1/ITS34_WP573_uploadable.pdf> 12 de diciembre de 2015.

Webster, Frank y Paul Bly (comps.) (1980), "The Demand for Public Transport: an International Collaboration Study”, Report, Transport and Road Research Laboratory, Crowthorne, Nueva Zelanda, pp. 54-62.

White, Peter (1981), "Travelcard tickets in urban public transport", Journal of Transport Economics and Policy, 15 (1), University of Bath, Bath, Inglaterra, pp. 17-34.

Recibido: 13 de febrero de 2017. Corregido: 28 de marzo de 2017. Aceptado: 26 de mayo de 2017.

Valentina Natividad-Viego. Doctora en economía por la Universidad Nacional del Sur, Bahía Blanca, Argentina. Actualmente es profesora adjunta del Departamento de Economía en la Universidad Nacional de Sur. Su línea de investigación actual es patrones de movilidad de pasajeros en ámbitos urbanos. Entre sus últimas publicaciones destacan: "Determinantes socioeconómicos y ambientales de la hipertensión arterial en la población adulta de Argentina", Revista Cubana de Salud Pública, 42 (1), Centro Nacional de Información de Ciencias Médicas, La Habana, Cuba, pp. 27-36 (2016); "Recent trends in occupational injuries and diseases in Argentina: a panel data approach", Occupational Diseases and Environmental Medicine, 3, Scientific Research Publishing, Wuhan, China, pp. 57-75 (2015); "Public policies for education completion: the case of 
Argentina's Fines Plan”, Education Policy Analysis Archives; 23 (116), Arizona State University, Phoenix, Estados Unidos, pp. 1-24 (2015); "Malnutrición: impacto de los hábitos y de las condiciones de vida en la población adulta urbana en Argentina", Revista de Ciencias de la Salud, 13 (2), Universidad del Rosario, Bogotá, Colombia, pp. 159-170 (2015).

Carolina Belen-Volonté. Maestranda en Economía de la Universidad Nacional Del Sur (UNS), Bahía Blanca, Argentina. Actualmente es miembro-investigadora del proyecto Movilidad intraurbana en la localidad de Bahía Blanca: modalidades y factores condicionantes. Su línea de investigación actual es patrones de movilidad de pasajeros en ámbitos urbanos. Entre sus últimas publicaciones destacan: "Eficiencia del transporte urbano de pasajeros en la localidad de Bahía Blanca (Argentina) 2007-2014", Semestre Económico, Universidad de Medellín, Medellín, Colombia (en prensa). 